\title{
REGISTRO DE STICHOSIPHON SANSIBARICUS (HIERON.) DROUET ET DAILY, CYANOPHYTA, EN LA FLORA CONTINENTAL. PERUANA.
}

REINA ZUUNIGAA.

\author{
Facultad de Ciencias Biológicas, Universidad Ricardo Palma. Apartado 138, Lima, Perú
}

\section{RESUMEN}

El género y especie estudiados, constituyen taxones poco conocidos para nuestra flora. Se reafirman sus características morfológicas y reproductivas, así como su habitat y distribución geográfica.

\section{SUMMARY}

The genus and species studied are taxa not well known in our flora. Complementary informations deals with its morphology, reproduction, habitat and geographical distribution.

\section{INTRODUCCION}

El análisis cualitativo de muestras de algas continentales procedentes de diferentes ambientes y localidades del país, nos da la oportunidad de registrar especies poco conocidas para nuestra flora, como es el caso de Stichosiphon sansibaricus (Hieron.) Drouet et Daily (=Stichosiphon regularis Geitler) miembro de las Clastidiaceae según Drouet et Daily (1956), o de las Dermocarpaceae del Orden Chamaesiphonales según Desikachary (1959).

Se dan a conocer las características morfológicas y reproductivas de esta especie epífita, se indica además su habitat y distribución geográfica.

\section{MATERIAL Y METODOS}

S. sansibaricus fue hallada en condición epífita en una muestra de Pithophora sp. procedente de un cuerpo de agua corriente en la ciudad de Bellavista, Departamento de San Martín.

Porciones del substrato vegetal fueron observadas al microscopio de luz en preparados temporales, previamente fijadas en una solución de formol al $5 \%$. La muestra estudiada se guarda en la Colección Ficológica del Herbario San Marcos (USM) del Museo de Historia Natural.

\section{RESULTADOS}

La clasificación de la especie estudiada se hace siguiendo a Desikachary (1959). Se dan a continuación las características más sobresalientes del Orden Chamaesiphonales, las familias y los géneros correspondientes.

Orden Chamaesiphonales. Comprende a formas unicelulares, gregarias, diferenciados en una porción basal y otra apical. Incluye a tres Familias: Cyanidiaceae, Dermocarpaceae y Chamaesiphonaceae.

Familia Dermocarpaceae. Incluye a formas unicelulares, solitarias o gregarias, diferenciadas en una porción basal y otra apical; epífitas. Se reproduce por endosporas.

\section{CLAVE PARA DIFERENCIAR LOS GENEROS}

1.Endosporas formadas por división del protoplasto en las tres dimensiones Dermocarpa

2. Endosporas formadas por división del protoplasto en una sola dimensión Stichosiphon

\section{Stichosiphon Geitler}

Stichosiphon sansibaricus (Hieron.) Drouet et Daily. Drouet et Daily 1952,10:223 ; 1956,125,fig.253 ; Desikachary 1959,176,pl.32,figs. 9-13, pl.33, figs.810 ; Drouet 1966,446 ; Bourrelly 1970,345 ,pl.9, figs.6-8. (Figs. 1,2)

Plantas microscópicas, elipsoidales a piriformes de 3 a 7 um de diámetro y hasta 40 um de alto; protoplasto azul-verde pálido, homogéneo; estuche delgado y hialino. 


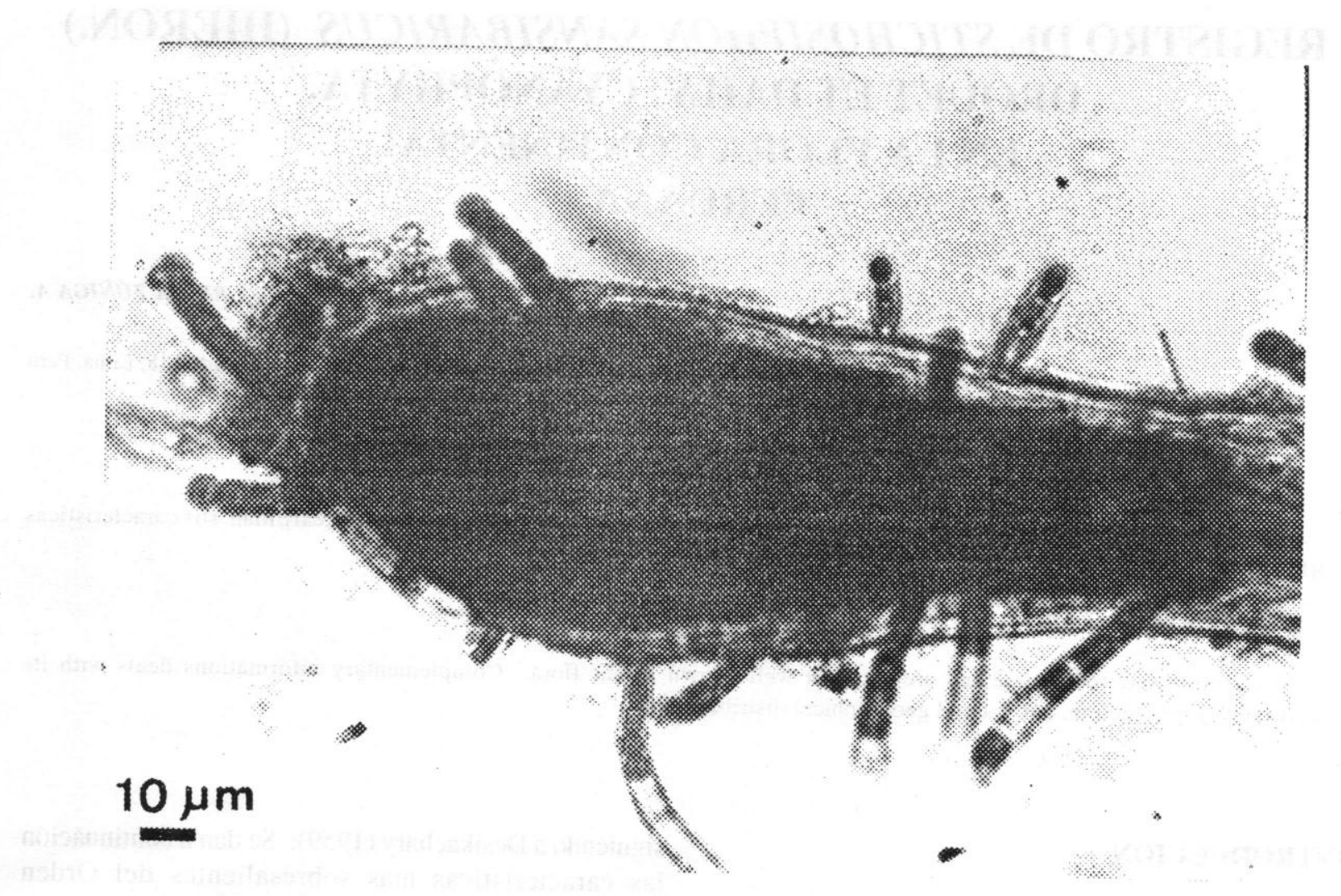

Fig.2 Varios individuos de Stichosiphon sansibaricus (Hieron.) Drouet et Daily, epífitos en la célula apical de
Pithophora sp.

Solitarias o gregarias, erguida o ligeramente curvada, epifítica por medio de una expansión mucilaginosa, con endosporas esféricas u ovoides en una serie lineal desde la base hasta el ápice.

Existen diversas opiniones relacionadas con la clasificación de este género y especie. Así, Drouet et Daily $(1952,1956)$ y Drouet $(1966)$ describieron a esta especie, perteneciente a la Familia Clastidiaceae del Orden Chamaesiphonales; Bourrelly (1970) la incluyó en la Familia Clastidiaceae (=Dermocarpaceae) del Orden Chamaesiphonales. Señaló que el género Dermocarpa está incluido en la Familia Hyellaceae del Orden Pleurocarpales. Las observaciones realizadas en los individuos de esta especie en relación con sus características morfológicas y formación de las endosporas, concuerdan con las de Desikachary (1959).

Distribución geográfica. Esta especie ha sido registrada en nuestro país en dos áreas geográficas relativamente próximas: en Bellavista, Departamento de San Martín y en la Quebrada de Puente Pérez, tributario del Huallaga, cerca a Tingo María, Departamento de Huánuco.

Esta es una especie rara y ha sido registrada en otras latitudes: Estados Unidos de Norte América, India, Filipinas, China, Zanzíbar. Drouet et Daily (1956) conside8

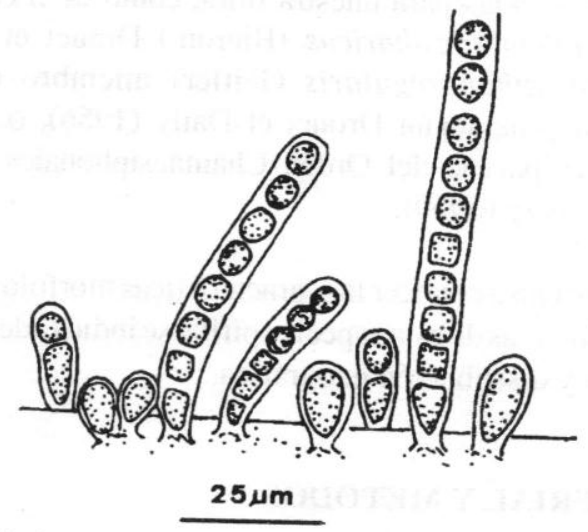

Fig.1 Individuos de Sticosiphon sansibaricus (Hiern.) Drouet et Daily, en estado vegetativo y formación de endosporas.

ran que la única especia válida es Stichosiphon sansibaricus (Hieron.) Drouet et Daily y las siguientes son basiónimo y sinónimos:

= Chamaesiphon sansibaricus Hieronymus, 1895

= Chamaesiphon filamentosa Ghose, 1924

= Chamaesiphon willei Gardner, 1927

= Stichosiphon regularis Geitler, 1932

=Stichosiphon filalmentosus (Ghose) Geitler, 1932

= Stichosiphon indicus Rao, 1935

= Stichosiphon indica var tenuis Gupta, 1950 


\section{AGRADECIMIENTO}

A María Maldonado, por la gentileza en la colecta de muestras diversas, en la que incluye a la especie estudiada.

\section{REFERENCIAS BIBLIOGRAFICAS}

BOURRELLY, P. 1970. Les algues d' eau douce. III. Les algues leues et rouges. Ed. N. Boubeé et Cíe. Paris.

DESIKACHARY, T.V. 1959. Cyanophyta. Indian Council of Agricultural Research, New Delhi.
DROUET, F. et W. DAILY, 1952. A synopsis of the Coccoid Myxophyceae. Butler University Studies 10: 120-223.

DROUET, F. et W. DAILY, 1956. Revision of the Coccoid Myxophyceae. Butler University Studies. 12: 1-218.

DROUET, F. 1966. The Catherwood Foundation PeruvianAmazon Expedition: Limnological and Systematics Studies. XIV. Cyanophyta. Monographs of the Academy of Natural Sciences of Philadelphia 14:445-448.

GEITLER, L. 1932. Cyanophyceae in Rabenhorst's Kryptogamenflora, Leipzig, 14:1196. 